\title{
Diferencias entre hombres y mujeres sobre las características del líder ideal en el ámbito laboral
}

\author{
Óscar Guerrero Rosado. Universidad de Granada \\ Azahara Valenzuela Luque. Universidad de Granada \\ Recepción: 24 de mayo de 2017 | Aceptado: 15 de diciembre de 2017 \\ Correspondencia: Óscar Guerrero Rosado | Correo-e: oscargr@correo.ugr.es
}

\section{iD 0000-0003-0108-6056}

Citar: Guerrero, O. y Valenzuela, A. (2017). Diferencias entre hombres y mujeres sobre las características del líder ideal en el ámbito laboral. ReiDoCrea, 6, 364-369.

\begin{abstract}
Resumen: Esta investigación se ha realizado con el fin de conocer las características estereotípicas que tanto hombres como mujeres asocian a su líder ideal y, además, analizar si esas características están asociadas a la preferencia de un líder hombre o mujer. Participaron 57 personas andaluzas que habían tenido una experiencia laboral mínima de 6 meses, con edades comprendidas entre los 20 y 30 años, y de las cuales 25 eran hombres (43.86\%) y 32 mujeres (56.14\%). En el estudio se utilizó una versión abreviada del Inventario de Bem (BSRI) para la recolección de los datos y el programa SPSS para su posterior análisis. Los resultados reflejan que los hombres asocian características estereotípicas andróginas a sus líderes ideales, en contraposición con las mujeres, las cuales optan por características femeninas. Por otro lado, existe una relación entre el sexo de los participantes y el del líder ideal, de forma que tanto hombres como mujeres prefieren líderes de su propio género.
\end{abstract}

Palabras clave: Estereotipo | Liderazgo

\section{Differences between men and women about ideal leader characteristics in the workplace}

\begin{abstract}
This investigation was carried out to determine stereotypical characteristics which men and women associate with their ideal leader and to analyze if these characteristics are related with preferences in the gender of a leader. The participants in this research study were Andalusians between 20 and 30 years old with at least 6 months work experience. Of the 57 participants, 25 were men (43.86\%) and 32 were women (56.14\%). In this study, a short version of the Bem Sex Role Inventory (BSRI) was used to collect the data and the SPSS program was utilized for the subsequent analysis. Results showed that men associate stereotypical androgynous characteristics to their ideal leaders, in comparison to women, who choose female characteristics. In addition, there is a connection between the sex of participants and the sex of the ideal leader in that both men and women preferred leaders of their own gender.
\end{abstract}

Keywords: Stereotypes | Leadership

\section{Introducción}

Educar en igualdad de género es una tarea primordial en la actualidad, ya que la construcción de un mundo de oportunidades equilibradas sin que el género sea un aspecto a tener en cuenta dependerá de los valores que hoy aprehendan nuestros niños y niñas. Teniendo en cuenta esto será necesario definir la terminología que permite llevar a cabo nuestra investigación con la que pretendemos aportar evidencia científica en el ámbito de la igualdad de género.

El concepto de género y las desigualdades que existen tienen gran impacto en nuestra sociedad por lo que desde finales del siglo $X X$ y a principios del $X X I$ se ha generado una constante investigación en este campo de estudio. Es Sherif en 1982 quien define el concepto de género como un esquema para la categorización social de los individuos. Este se diferencia del concepto sexo en que hace referencia a la parte social y cultural, sin tener en cuenta la parte biológica. El género da lugar a la categorización de las personas en nuestra sociedad actual, provocando así diferencias 
entre ambos, no solo por sus apariencias físicas, sino también respecto a sus actitudes, conductas y roles que adoptan.

Estas diferencias se relaciona con la formación y aceptación de los estereotipos de género, los cuales se definen como un conjunto de creencias que han sido establecidas y aceptadas social y culturalmente con el paso del tiempo respecto a los hombres y mujeres (Bem, 1974; López-Sáez, 1993). Estos pretenden incluir todas las características físicas y psicológicas prototípicas de ambas categorías, siendo así características psicosociales prototípicas. La Teoría de los Esquemas de Género propuesta por Bem (1981) sostiene que el contenido social de los esquemas ejerce una influencia sobre el procesamiento de la información relacionada con el género, es decir, que los esquemas previos de la persona van a influir tanto sobre el procesamiento de la información como sobre las conductas estereotipadas, de forma que ayudarán o no a mantener los estereotipos de género.

Al afirmar que el género se construye culturalmente, se defiende que el origen de las características es situacional, lo que da lugar a los estereotipos, los cuales simplifican el procesamiento de la información, mantiene el status quo resistiendo al cambio, a la misma vez que determinan lo que es normal o aceptable, y lo que se desvía de la norma social (Cuadrado, Gaviria y López-Sáez, 2009). De este modo, será la cultura específica de cada grupo la que connote y cargue de contenidos el género.

En la actualidad se observa cómo el género es un distribuidor dispar de oportunidades que favorece la desigualdad y diferencias entre géneros. Concretamente en el ámbito laboral, podemos observar como cada año los porcentajes de ocupación laboral de hombres y mujeres muestran una proporción más equilibrada, apuntando a la igualdad de género. En concreto, en el año 2015 el 45,4\% de la población española ocupada fueron mujeres; pero a pesar de ello, no existe este mismo equilibrio en los porcentajes de ocupación en puestos de directivos y gerentes, ya que más del doble de estos puestos son ocupados por hombres. No obstante, también se observa una orientación hacia la igualdad de género ya que cada año la proporción de mujeres directivas y gerentes aumenta pero, teniendo en cuenta que en 2014 el porcentaje fue de $30.88 \%$ y que en 2015 aumentó a $31.37 \%$, podemos decir que este progreso es muy discreto (Instituto de la Mujer).

Con motivo de dar explicación a la desigualdad de género en cuanto al liderazgo se refiere, se ha generado multitud de investigación.

Por un lado, estos datos desfavorables para la mujer podría explicarse por una diferencia en el tipo de liderazgo que ejerce, siendo este menos efectivo que el ejercido por el hombre. Desde la investigación tradicional, la cual es importante tener en cuenta ya que expone la base cultural de la situación actual, se han asociado diferentes rasgos y valores en función del género. En 1955, Parson y Bales observaron que al hombre se le asigna un papel instrumental orientado a metas, mientras que a la mujer un papel expresivo orientado a las relaciones personales.

Además, Bakan (1966), expone una diferencia de intereses: comunales en la mujer e individualistas en el hombre.

Estas aportaciones están en consonancia con el meta-análisis elaborado por Eagly y Johnson (1990) que expone cómo las mujeres lideran con estilos más democráticos y participativos, mientras que los hombres lideran de forma más autocrática y directiva. Por otro lado, en este mismo meta-análisis no se evidencian diferencias significativas en los estilos de orientación hacia la tarea o hacia las relaciones. 
Sin embargo, en 2003 un estudio de Cuadrado, Molero y Navas con población española, y apoyado por los resultados de otro estudio de Cuadrado en 2004, informó que tanto hombres como mujeres líderes perciben que adoptan estilos de liderazgos similares; tanto en la dimensión democrático-autocrático, lo que no coincide con el meta-análisis de Eagly y Johnson, como en la de orientación a la tarea o a las relaciones. Aunque sí se observó diferencias en las relaciones entre los estilos de liderazgo: las mujeres líderes relacionan un buen estilo de liderazgo (liderazgo transformacional) con estilos autocráticos y orientados a la tarea.

En esta evolución en los datos aportados por las diferentes investigaciones, en cuanto a la diferencia de los estilos de liderazgo en función del género, no solo influye el cambio cultural que está ocurriendo constantemente en nuestra sociedad orientado a la igualdad de género, sino que también hay que tener en cuenta que los distintos métodos de investigación han podido influir sobre los resultados. Aun así, no parece que una diferencia en los estilos de liderazgo pueda constituir un deficiente liderazgo de las mujeres como explicación de la desigualdad hacia ellas de la ocupación de puestos de dirección.

Otra propuesta explicativa de la dificultad que experimentan las mujeres para acceder a puestos de dirección es la teoría del Techo de Cristal, la cual propone que existen prejuicios implícitos y desconfianza en las mujeres para confiarles puestos de responsabilidad, y por ello su ascenso laboral se estanca una vez llegado a ciertos límites. Estos límites parece que han ido desapareciendo y que la situación ha empezado a cambiar; por lo que el acceso de la mujer al liderazgo, aún poco frecuente, ha forzado una actualización de esta teoría. Eagly y Carli (2007) proponen reemplazar la metáfora del Techo de Cristal por la metáfora del Laberinto, en la que la mujer no tendría barreras que impidan el acceso a cargos de dirección, pero sí que su ascenso a estos puestos implicaría recorrer un camino más difícil y sortear obstáculos con los que los hombres no tienen que lidiar.

La teoría de congruencia del rol hacia las mujeres líderes, propuesta por Eagly y Karau (2002), da una explicación a aquellos límites, obstáculos y dificultades que proponen las dos metáforas mencionadas anteriormente. Estos se deberían a prejuicios generados por la incongruencia de las características estereotípicas femeninas con los atributos requeridos para el rol de líder, tradicionalmente asociados a características estereotípicas masculinas. Esto ha forzado a la mujer a adoptar, además de características femeninas, características masculinas llegando a describirse a sí mismas con atributos andróginos (de ambos géneros), mientras que los hombres se definen a sí mismos en función de características agénticas como dominancia, personalidad fuerte o dureza (Castro y Lupano, 2010).

Tras observar la relevancia del género en el liderazgo, en la presente investigación se tratará de conocer las características que hombres y mujeres asocian a su líder ideal, a la vez de observar si hay congruencia entre las preferencias de hombres y mujeres, respecto al género del líder que prefieren, y las características que previamente han asociado a su líder ideal.

En base a investigaciones previas cabe esperar que los hombres definan a su líder ideal con características estereotípicas masculinas, en contraposición a las mujeres que van a asociar características andróginas a su líder ideal. Además, ambos géneros, hombres y mujeres van a preferir líderes hombres, aunque las mujeres lo harán con una intensidad menor. 


\section{Método}

\section{Participantes}

La investigación se realizó con un grupo de 57 personas andaluzas que participaron de forma voluntaria, 25 hombres $(43,86 \%)$ y 32 mujeres $(56,14 \%)$, con edades comprendidas entre los 20 y 30 años, y con requisito común haber tenido al menos una experiencia laboral mínima de 6 meses.

\section{Materiales}

Se ha utilizado una versión corta del Inventario de Roles de Sexo de Bem (BSRI) compuesta por los 18 rasgos que resultaron ser 9 estereotípicamente masculinos y 9 estereotípicamente femeninos (Bem, 1974; Martínez, Navarro, Yubero, 2009). Este instrumento se utiliza para medir el grado de identidad de rol de género que se han relacionado con los términos de masculinidad y feminidad de hombres y mujeres (López Zafra y López-Sáez, 2001). La lista de características mostradas a los participantes tenían un formato Likert de respuesta con 7 opciones, donde 1 corresponde a nunca o a casi nunca y 7 a siempre o a casi siempre. Concretamente, muestra dos dimensiones de 9 ítems cada una, como son masculinidad (instrumentalidad) y feminidad (expresividad) y cada participante debe señalar el grado en que la característica presentada representaría a su líder ideal. Según este instrumento, los resultados obtenidos permiten clasificar a los participantes en cuatro categorías como son masculino (sujetos que describen a su líder con atributos prototípicamente masculinos o agentes), femenino (participantes que describen a su líder con atributos prototípicamente femeninos o comunales), andrógino (sujetos que describen a su líder con características prototípicas tanto comunales como agénticas) e indiferenciado ( personas que describen a su líder débilmente con características de ambos géneros) (Castro y Lupano, 2010).

\section{Procedimiento}

El estudio siguió un proceso común para todos los participantes que consistió en administrar la versión corta del BSRI a través de internet, concretamente por una encuesta de google realizada por los investigadores con el fin de facilitar la obtención de la muestra y tener los resultados de forma directa. Se les pidió a los sujetos que imaginaran a su líder ideal independientemente del género cuando respondiesen a las preguntas. Al final, en otra página para evitar que los participantes lo viesen con antelación, se les preguntó si prefieren un líder hombre o mujer y con qué intensidad (escala Likert de 6 opciones desde una intensidad muy baja hasta una muy alta). Tras obtener los resultados de la encuesta de google, los datos se analizaron mediante el programa informático IBM SPSS statistics 23.

\section{Resultados}

Tras la aplicación del inventario de Bem se observa que no hay diferencia significativa entre hombres y mujeres a la hora de responder los ítems femeninos, con una media de 4.42 en hombres y 4.68 en mujeres. Sin embargo, esta diferencia sí es significativa al escoger ítems masculinos $(p=.03)$, con medias de 4.19 en hombres y 3.82 en mujeres. Observando estos datos se puede concluir que las mujeres han optado por un perfil femenino y los hombres por uno andrógino. Estos resultados evidencian que no se cumple la primera hipótesis, en la cual se plantea que las mujeres asociarían características andróginas al líder ideal, mientras que los hombres asociarían características masculinas. 
Por otro lado, sí se han encontrado diferencias significativas en la relación entre el sexo de los participantes y el del líder ideal $(p=.031)$. Concretamente, el $67 \%$ de los hombres han elegido a un hombre como líder ideal, y el $63 \%$ de las mujeres han preferido un líder mujer. Sin embargo, tanto en hombres como en mujeres no es significativa la correlación entre la respuesta de los los ítems que caracterizan al líder ideal (masculinos o femeninos) y el sexo del líder que prefieren. Además la intensidad de preferencia por un líder hombre o mujer, ha sido muy baja (1.96 hombres y 2.15 mujeres), lo cual puede ser debido a la deseabilidad social.

\section{Discusión}

El presente estudio se llevó a cabo con objeto de conocer las características que los seguidores atribuyen a su líder ideal y comprobar si estas eran congruentes con el sexo elegido para éste. Según en los resultados obtenidos se puede ver cómo las características ideales para un líder, desde el criterio de un seguidor, va a depender del género de este último ya que los hombres optan por un perfil andrógino y las mujeres por un perfil femenino. Sin embargo esta evidencia no concuerda con el perfil que se atribuyen los propios líderes, lo que abre un debate hacia qué perfil de líder es el más apropiado y eficaz.

Además, los datos evidencian que no solo las características del líder ideal van a depender del género del seguidor, sino que también existen diferencias por la preferencia del género del líder siendo un líder hombre para los hombres y una líder mujer para las mujeres. Por otro lado, al responder cómo de importante es el género del líder para ellos, los seguidores dicen que es un aspecto de baja relevancia. Este último dato, puede que esté sesgado por la deseabilidad social ya que no concuerda con la diferencia de preferencia.

En cuanto a las limitaciones de esta investigación se debe tener en cuenta que se ha realizado con una muestra reducida de 57 participantes andaluces de entre 20 y 30 años, los cuales, aunque nos proporcionan unos resultados orientativos no consideramos que la muestra sea apta para generalizar los datos a la población española. Además, las pruebas psicométricas fueron administradas online, lo que por un lado generalmente aporta intimidad y tranquilidad al participante, pero por otro no permite mantener un mismo contexto para todos ellos.

También se cree conveniente que el cuestionario utilizado, el Inventario de Roles de Sexo de Bem, sea actualizado ya que desde 1974 hasta nuestros días las características asociadas a distintos sexos pueden que hayan cambiado considerablemente. No obstante, se considera que, aunque distintas características aún se asocien a distintos sexos según su prevalencia en ellos, es una interpretación inadecuada de los resultados y un obstáculo para la igualdad de género considerar que un líder eficaz tiene ciertas características masculinas o femeninas. En contraposición se propone considerar que ciertas características son propias de un líder óptimo, sin aludir su relación con los distintos géneros.

Cómo líneas de investigaciones futuras se considera necesario constatar los datos del presente estudio con una muestra más amplia, que además incluya participantes de distintas edades para conocer si existen diferencias entre distintos grupos de edad y así observar cómo la sociedad avanza en dirección a la igualdad de género en cuanto al liderazgo de la mujer se refiere. También, sería interesante adaptar esta investigación para conocer qué tipos de líderes prefieren los niños y niñas, especialmente en los ámbitos educativos y familiar, de modo que nos permita adaptar nuestro rol de líderes a sus exigencias y trabajar las posibles preferencias desiguales 
de género si existiera. Además, nos preocupa el papel que toma la deseabilidad social en los participantes a la hora de responder ciertos ítems comprometidos como por ejemplo: ¿Con qué intensidad prefieres que tu líder ideal sea hombre o mujer?

Para finalizar, se invita al debate y reflexión sobre la evidencia de que ambos sexos tienen preferencias por distintas características en un líder ideal, lo cual implica que el trato que ellos exigen es distinto, pero ¿Estaríamos luchando por la igualdad de género si los líderes prestan un trato diferencial a sus seguidores en función del sexo?

Referencias

Bakan D. (1966). The duality of human existence. Chicago, C.A.: Rand McNally.

Bem S.L. (1974). The measurement of psychological androgyny. Journal of Consulting and Clinical Psychology, 42(2), $155-162$.

Bem S.L. (1981). Gender Schema Theory: A cognitive account of sex typing. Psychological Review, 88(4), 354-364.

Castro A. y Lupano M.L. (2010). Análisis de características estereotípicas de género en líderes y seguidores. SUMMA Psicológica UST, 7(2), 5566.

Cuadrado, I. (2004). Valores y rasgos estereotípicos de género de mujeres líderes. Psicothema, 16(2), 270- 275.

Cuadrado I., Gaviria E. y López-Sáez M.(2009). Introducción a la Psicología Social. Madrid: Sanz y Torres.

Cuadrado, I., Molero, F. y Navas, M. (2003). El liderazgo de hombres y mujeres: Diferencias en estilos de liderazgo, relaciones entre los estilos y predictores de variables de resultado organizacional. Acción psicológica, 2(2), 115-129.

Eagly, A. H., \& Carli, L. (2007). Through the labyrinth. The truth about how women become leaders. Boston: Harvard Business School Press.

Eagly, A. H. y Karau, S. J. (2002). Role congruity theory of prejudice toward female leaders. Psychological Review, 109(3), 573-598.

Eagly, A. H. y Johnson, B. T. (1990). Gender and leadership style: A metaanalysis. Psychological Bulletin, 108, $233-256$.

Instituto de la Mujer (2015). Mujeres en cifras. Recuperado de www.inmujer.gob.es/MujerCifras/Home.htm.

Martínez I., Navarro R., Yubero S. (2009). Estereotipos de género entre los adolescentes españoles: imagen prototípica de hombres y mujeres e imagen de uno mismo. Información Psicológica, 95, 77-88.

López-Sáez M. (1993). Tipicidad de identidad de género y comparación intergrupal. Revista de Psicología Social, 8(2), $189-200$.

López-Zafra, E. y López-Sáez, M. (2001). Por qué las mujeres se consideran más o menos femeninas y los hombres más o menos masculinos. Explicaciones sobre su autoconcepto de identidad de género. Revista de Psicología Social, 16, 193-207.

Parson T. \& Bales, R.F. (1955). Family, socialization and interaction process. New York: Free Press.

Sherif C.W. (1982). Needed concepts in the Study of Gender Identity. Psychology of Women Quarterly, 6(4), 375-398. 